Castaño, C., Zamarra, J., y Salazar, E. (2021). El control interno y las tecnologías de la información en las Mipymes del oriente antioqueño: estado actual de su implementación y oportunidades de mejora. Contaduría Universidad de Antioquia, 78, 75-104. Doi: https://doi.org/10.17533/udea.rc.n78a03

\title{
El control interno y las tecnologías de la información en las MIPYMES del oriente antioqueño: estado actual de su implementación y oportunidades de mejora
}

Carlos Eduardo Castaño Ríos

Universidad de Antioquia eduardo.castano@udea.edu.co Orcid: 0000-0003-4117-0375

Julián Esteban Zamarra Londoño

Universidad de Antioquia julian.zamarra@udea.edu.co Orcid: 0000-0003-0539-4187

Erika Janeth Salazar Jiménez

Universidad de Antioquia janeth.salazar@udea.edu.co Orcid: 0000-0002-1402-5845 
Castaño, C., Zamarra, J., y Salazar, E. El control interno y las tecnologías de la información en las Mipymes...

El control interno y las tecnologías de la información en las MIPYMES del oriente antioqueño: estado actual de su implementación y oportunidades de mejora

Resumen: Las MIPYMES son un componente fundamental de la cadena empresarial en el mundo y en estas se han hecho necesaria la implementación de procesos de control que garanticen la continuidad de esta en el tiempo. El objetivo de este texto es presentar algunos asuntos del control interno contable como resultado de la aplicación de una encuesta en una muestra de 379 MIPYMES del oriente antioqueño colombiano en el marco de un proyecto de caracterización frente a la gestión contable y financiera de estas entidades. Los resultados evidencian que existen diversos problemas en las MIPYMES de la región, a causa de que sus administradores, quienes en muchos casos son los mismos dueños, desconocen herramientas de gestión y control que permitan realizar una mejor organización que permita la continuidad de sus negocios en el tiempo.

Palabras clave: MIPYMES, gestión financiera, control interno.

Internal control and information technologies in micro and SMEs from east Antioquia: current state in their implementation and opportunities for improvement

Abstract: Micro and SMEs are a fundamental component of the business chain in the world and it has been made necessary to implement in them control processes guaranteeing their permanence in time. The aim of this article is to present some internal accounting control issues resulting from the application of a survey to a sample of 379 micro and SMEs from east Antioquia, Colombia, in the frame of a characterization project about the accounting and financial management of these entities. The results evidence diverse problems in the micro and SMEs of the region caused by their administrators, who are the owners in many cases, and who ignore the management and control tools which allow to achieve a better organization that enables the permanence of their business in time.

Keywords: Micro and SMEs, financial management, internal control.

O controle interno e as tecnologias da informação nas MPME do leste de Antioquia: estado atual de sua implementação e oportunidades de melhoria

Resumo: As MPME são um componente fundamental da cadeia empresarial no mundo e nestas tem se feito necessária a implementação de processos de controle que garantam a continuidade desta no tempo. O objetivo deste texto é apresentar alguns assuntos do controle interno contábil como resultado da aplicação de uma enquete em uma amostra de 379 MPME do leste de Antioquia (Colômbia) no marco de um projeto de caracterização frente à gestão contábil e financeira destas entidades. Os resultados evidenciam que existem diversos problemas nas MPME da região, a causa de que seus administradores, quem em muitos casos são os mesmos donos, desconhecem ferramentas de gestão e controle com as quais possam realizar uma melhor organização que permita a continuidade de seus negócios no tempo.

Palavras chave: $M P M E$, gestão financeira, controle interno.

Le contrôle interne et les technologies de l'information dans les PME de l'Orient du département d'Antioquia : État actuel de leur mise en œuvre et possibilités d'amélioration

Résumé: Les MPME sont une composante essentielle de la chaîne d'entreprises dans le monde et il est devenu nécessaire de mettre en place des processus de contrôle qui garantissent la continuité de celle-ci dans le temps. Les résultats montrent que les MPME de la région rencontrent des problèmes divers du fait que leurs gérants, qui sont souvent aussi leurs propriétaires, ne disposent pas d'outils de gestion et de contrôle leur permettant de mener une meilleure organisation qui puisse donner lieu à la continuité de leurs activités dans le temps.

Mots-clés: MPME, gestion des finances, contrôle interne 


\title{
El control interno y las tecnologías de la información en las MIPYMES del oriente antioqueño: estado actual de su implementación y oportunidades de mejora
}

\author{
Carlos Eduardo Castaño Ríos, Julián Esteban Zamarra Londoño y Erika Janeth Sala- \\ zar Jiménez
}

https://doi.org/10.17533/udea.rc.n78a03

Primera versión recibida en julio de 2020 - Versión aceptada en noviembre de 2020

\section{Introducción}

Las micro, pequeñas y medianas empresas - MIPYMES - son parte fundamental de la mayoría de las economías en el mundo, pues a pesar de las limitaciones propias a causa de su tamaño y otros problemas derivados de su gestión, estas organizaciones contribuyen con aproximadamente $40 \%$ del PIB en un país como Colombia y propician espacios y plazas equivalentes a un poco más del 60\% del empleo del país (Castaño y Zamarra, 2012; La República, 2019; Arango, 2019). No obstante, una gran problemática que se ha evidenciado en su accionar, tiene que ver con la falta de planeación y el escaso seguimiento a sus recursos con herramientas que están al alcance de los empresarios y que se ofrecen desde el mundo de la contabilidad, tales como, variadas metodologías de control que contribuyen a la eficacia operativa de las actividades de la entidad (Correa, Ramírez y Castaño, 2010; Castaño, Zamarra y Salazar, 2017).

El presente artículo surge como resultado de la investigación desarrollada en el oriente antioqueño, una de las 9 subregiones de Antioquia, que cuenta con 26.667 empresas inscritas en la Cámara de Comercio del Oriente Antioqueño (CCOA) para el año 2015. De dichas entidades, 99,59\% son MIPYMES, ubicando a esta subregión como la segunda del departamento de Antioquia en cuanto a su representatividad económica, después del Valle de Aburrá que comprende la ciudad capital y municipios cercanos (Cámara de Comercio del Oriente Antioqueño, 2015). 
Castaño, C., Zamarra, J., y Salazar, E. El control interno y las tecnologías de la información en las Mipymes...

La región del oriente antioqueño es de vital importancia en el desarrollo del departamento de Antioquia, toda vez que su cercanía al área metropolitana del Valle de Aburrá, la presencia de una zona franca, del aeropuerto internacional, así como todo un conjunto de infraestructura vial de alta representatividad, proyectan la zona en crecimiento económico permanente, con miras a la exportación de todo tipo de bienes y servicios. Es así como surge para estas empresas y para sus propietarios la necesidad de implementar herramientas de gestión de alta calidad y mejores estructuras desde el punto de vista financiero y contable que comprenden, además, medidas de control interno y el uso de las tecnologías para aumentar la efectividad de los procesos.

Estas relaciones entre los sistemas de información y los modelos contables y de control interno de la entidad, propenden por la efectividad de la misma y potencian las capacidades de este tipo de organizaciones centrándose en que no solo se oferten productos y servicios innovadores o de amplia necesidad para cierto tipo de población, sino que se requiere también que estas empresas sean viables en el largo plazo, es ahí donde radica la importancia de realizar un acercamiento y caracterización de dichas organizaciones en torno a sus prácticas de gestión y encaminar acciones que conlleven a su mejoramiento continuo desde distintas perspectivas (Balcazar, 2018), en este caso, desde el punto de vista del control interno y qué herramientas de tecnologías de la información usan en relación a las prácticas de control interno.

El foco de atención y objetivo de este trabajo es presentar diversos aspectos sobre las características del control interno contable en el marco de un proyecto sobre caracterización de la gestión contable y financiera de las MIPYMES del oriente del departamento de Antioquia (Colombia), para lo cual se indagó en una muestra de 379 MIPYMES de la región sobre aspectos propios de la gerencia del sistema de información contable y el análisis para la toma de decisiones. Desde el punto de vista metodológico, el principal instrumento de investigación fue la encuesta, mediante la que se realizaron indagaciones a propietarios y/o administradores de las empresas de la muestra y se analizaron las respuestas obtenidas. Dichos análisis guardan relación con estudios de tipo exploratorio, dado que se tomaron las respuestas y se tabularon los resultados para conocer los grados de avance en distintos frentes de trabajo desde la gestión financiera $\mathrm{y}$, en particular, este texto presenta los asuntos vinculados al proceso de control de los recursos organizacionales, en especial, enfocado en lo que se denomina control contable.

Las respuestas indican que los sistemas de control interno son escasos y a la vez precarios en esta muestra empresarial y que en aquellas entidades en las que se tienen establecidos procedimientos de control, estos recaen en su mayoría sobre procesos de compras y ventas, que se ven reflejados en los inventarios de las compañías. El aprovechamiento de tecnologías tampoco es una constante 
y representan oportunidades de mejora para la gestión de estas en la medida que ellas permiten automatizar controles y facilitan procesos de gestión.

El texto presenta en primer lugar los antecedentes de investigación en torno a los sistemas de control interno en entidades pequeñas y medianas, sus ventajas y oportunidades para la gestión y mejora continua, al mismo tiempo que los relaciona con las herramientas tecnológicas que facilitan o mejoran dichas gestiones. Posteriormente, se presentan los aspectos metodológicos que guiaron la investigación para el análisis de los resultados obtenidos y finalmente se encuentran los resultados y conclusiones del estudio en torno al control interno y las tecnologías de la información en la MIPYMES.

\section{Antecedentes}

El proceso de globalización cada vez más acelerado al que se enfrenta el mundo en la actualidad, ha llevado a las organizaciones a un nuevo macroescenario que puede llegar a ser más amplio de lo que hoy se cree y que afecta considerablemente a las MIPYMES de cualquier región o país. En este sentido, este proceso de globalización genera que dichas empresas, independiente de su tamaño, vean la necesidad de tener herramientas de gestión contables y financieras que conlleven a un mejor uso de los recursos, estableciendo procedimientos que den respuesta a los cambios en búsqueda de que la compañía se mantenga competitivamente en su entorno (Alonso y otros, 2016; López y otros, 2016) y generen valor agregado (Rivera-Godoy y Rendón-Perea, 2019). De esta manera, el mundo contemporáneo exige la existencia de controles que minimicen los riesgos a que están expuestas las organizaciones sin importar su tamaño o sector económico, de forma tal que las medidas que se aplican deben funcionar en cualquier momento y aportar a su mejoramiento continuo desde el punto de vista operacional y financiero, de manera que propicien una acertada toma de decisiones para la continuidad de las organizaciones (Pereira, 2015). Además, no se puede dejar de lado la importancia de una gestión ética y responsable de las organizaciones con el objetivo de proteger el interés general sobre el particular en procura de una sostenibilidad futura de la humanidad (Fuentes- Doria y otros, 2019).

Ahora bien, en vista de las necesidades de una mejor gestión del control, se ha generado el interés de mantener mecanismos que permitan tener una mayor confiabilidad y vigilancia sobre las actividades y procesos que llevan a cabo las organizaciones y que salvaguarden el patrimonio de los propietarios, al mismo tiempo que contribuyan al mejoramiento de índices de desempeño y calidad que terminan en la optimización y racionalización de las decisiones de los usuarios de la información contable que genere la MIPYME.

Lo anterior indica que los sistemas de control que apuntan al mejoramiento de los procesos organizacionales tomen gran relevancia en la toma de decisiones. 
De acuerdo con Henri (2006) una gran parte de la literatura ha estado enfocada a la dependencia que tiene cualquier sistema de control en una cultura de control fundamentada y fuerte en la que todos los miembros de la empresa contribuyan con dicho propósito y se autoevalúen respecto a ello. Con esto, queda claro que el uso de adecuados controles, que sean efectivos y que operen de manera coherente con el desarrollo de las actividades organizacionales, se convierte en una ventaja competitiva que coadyuva junto con otras variables de gestión al éxito de una organización sobre su competencia y más aún en el caso de las MIPYMES, entre las que suele existir un menor grado de diferenciación por sí solo (Barney, 1991) (Sonrensen, 2002).

No obstante, y pese a que son claras las grandes ventajas que tienen los controles en la organización, ha sido amplia la investigación que ha puesto en evidencia el hecho de que las MIPYMES en general no tienen adecuados sistemas de control interno o en su defecto ni siquiera tienen alguno diseñado y mucho menos implementado (Choe, 1996; Li, Liu, Duan y Li, 2008; Aguirre y Armenta, 2012). En contraste, algunos otros autores del contexto local como Gómez et al. (2017), han definido que si bien puede existir una cantidad representativa de empresas en las que se tengan controles establecidos de manera informal, los mismos solo funcionan ante fenómenos o coyunturas de la entidad, lo que no permite afianzar una cultura de control que optimice las posibilidades y oportunidades de mejora que ofrecen dichos sistemas. Esto quiere decir que los controles que operan en las MIPYMES son principalmente de carácter correctivo y pocos son aquellos que se establecen con miras a la prevención de errores o fraudes representativos por parte de colaboradores o incluso de los mismos propietarios, de forma tal que le eviten a la administración desgastes o reprocesos innecesarios.

Estudios previos se han enfocado en las posibles relaciones que pueden existir entre las empresas de pequeño y mediano tamaño y el uso de adecuados controles internos y de reportes de seguimiento financiero y operacional para la autoevaluación continua (Bardhan, Lin y Wu, 2015; Doyle, Ge y McVay, 2007). Sin embargo, pocos han hecho un relacionamiento entre la aplicación de diferentes variables que se asocien a la calidad del control interno, como por ejemplo las particularidades de la administración de este tipo de entidades o la relación de los controles con su forma más o menos manual de aplicación. La ausencia de sistemas de control efectivos se ha convertido en un problema particularmente común en las MIPYMES y que las hace mucho más vulnerables a los efectos del entorno y a los errores internos que puedan pasar por desapercibidos, sean o no con intencionalidad (Saha y Mondal, 2012).

Dado que en la mayoría de los casos las MIPYMES, sobre todo cuando son familiares (European Commission, 2009), suelen tener limitaciones para la ejecución de gestiones integrales y presentan mayores retos que en una compañía 
de gran tamaño pues no dispone de recursos, especialmente de asesoría especializada en todas las áreas, para dar respuesta al cambiante entorno y a las nuevas necesidades de la sociedad. De acuerdo con Deshmukh (2004) existen en el contexto de las pequeñas y medianas empresas cuatro causas principales para que no alcancen los objetivos que se han propuesto al surgir: errores humanos, ausencia de factores de gestión, colaboradores que sobrepasan los controles implementados y gerentes que también sobrepasan los controles para presentar informes financieros más llamativos.

Al respecto, es válido analizar la influencia que existe en este tipo de organizaciones, las maneras de administración y más aún sobre quienes desempeñan la administración en sí misma que son, en la mayoría de los casos, los dueños de la empresa, además, de contar con estructuras bastante informales (Giraldo y Sinisterra, 2018). Por ejemplo, Lozano (2015) esboza la relevancia de algunas particularidades como el conservadurismo, las decisiones de corto plazo y el uso del efectivo generado en la organización que desfavorecen la permanencia de la entidad en el tiempo y propician escenarios de iliquidez o desactualización de la empresa respecto a la situación del mercado. Adicionalmente, la inclusión de aspectos del entorno familiar en las decisiones de dirección de la empresa genera otras distorsiones a los objetivos de esta que si bien pueden no representar amenazas de alta significancia sí podrían generar problemáticas internas (Gómez, Cruz, Berrone y De Castro, 2011).

Otras investigaciones han denotado la falta de apropiación de buenas prácticas de control interno por parte de la dirección de las MIPYMES, debido a un exceso de confianza sobre las personas que dirigen la entidad (Leenders y Waarts, 2003). Otros estudios han demostrado que la orientación familiar en la dirección tiene relación negativa con el establecimiento de mecanismos de contabilidad y control, bajo la premisa de la no necesidad de controles sobre los mismos dueños de la empresa o la ausencia de planes de gestión macro dentro de los cuales se destinen recursos para dichos sistemas de control (Speckbacher y Wentges, 2012).

Por otro lado, existen algunos autores que han determinado un relacionamiento positivo entre los sistemas de gobierno que establecen los propietarios de la entidad en sus empresas, puesto que el círculo interno de dirección desarrolla, según su estilo propio, papeles cruciales en la legitimación del sistema de control que se instaura (Efferin y Hartono, 2015). Dicho sistema está basado en el concepto como tal de familia y la confianza interna que se guardan entre los integrantes de esta.

La literatura ha puesto en evidencia, además, la premisa de que a nivel de las micro y pequeñas empresas no existe un nivel de confianza hacia los servicios de asesoría que pueden ofrecer los profesionales en dichas organizaciones (Cesaroni y Sensuti, 2017). Al respecto, es válido afirmar que se ha dado cierta 
Castaño, C., Zamarra, J., y Salazar, E. El control interno y las tecnologías de la información en las Mipymes...

discrepancia entre las prácticas contables de los administradores de este tipo de entidades y los profesionales contables, puesto que los contadores llegan a estos entornos laborales con la intención de no interferir en las relaciones familiares, puesto que los contadores están principalmente preocupados por problemas técnicos de su profesión y no muestran interés en la interacción y la comunicación con los miembros de la familia es escasa (Romero, Melgarejo y Vera, 2015; González, Viga y Fierro, 2017). Esto, en suma, puede causar una discrepancia entre la práctica profesional del contador y las expectativas de la familia sobre su rol en la empresa como mediador para la resolución de diversos tipos de problemáticas. Así pues, todos los factores anteriores se relacionan con la ineficiencia de un sistema de control interno que, si bien puede ser básico, debería garantizar la razonabilidad de las operaciones que se desarrollan en la entidad y propender por mejorar el entorno y la competitividad de las organizaciones que hacen un buen uso de dichos sistemas, que pueden, además, estar integrados con herramientas tecnológicas.

Un argumento habitual para la justificación de la no existencia de controles se da respecto a la falta de recursos humanos o financieros para que su efectividad sea asegurada (Fernández y Nieto, 2005). En general, el uso de herramientas tecnológicas tiene una relación directa con el crecimiento de las organizaciones, por ello las MIPYMES han manifestado que la ausencia de personas dentro de su estructura que dominen los conocimientos administrativos suficientes para dirigir y controlar una organización impacta en su desempeño. En la medida en que incrementen el uso de herramientas tecnológicas, estas empresas podrán a su vez incentivarse a sí mismas a propender por una mejor gestión basada en el control no solo de los procesos, personal o departamentos (elementos internos), sino también de clientes, proveedores y Estado (elementos externos).

Por ejemplo, solo 5\% de las pequeñas y medianas empresas que existen en Colombia utilizan masivamente las TICs para desarrollar sus negocios; lo que traduce que la mayoría de los empresarios no se relacionan con ninguno de los usuarios externos de su información mediante herramientas informáticas que les faciliten canales de interacción como la internet o las bases de datos (Forseilles, 2013).

Todas estas oportunidades de mejora inciden en que las MIPYMES tengan un alto grado de vulnerabilidad ante los factores externos a la entidad. Dado que, tal y como se ha mencionado, las problemáticas a las que se enfrentan las organizaciones no solo provienen de ellas mismas. El nuevo contexto demanda que todas las empresas se concentren tanto en los aspectos internos como los externos que puedan tener influencia en la estructura organizacional. Esto porque las MIPYMES tienden a enfocar su gestión basadas en lo que hace la competencia o al curso de los negocios de empresas líderes del sector (Monge, Alfaro y Alfaro, 2005; Gómez, Villarreal y Villarreal, 2015)). 
Ello toma relevancia en la medida que pueden descuidarse aspectos internos de la entidad, sacrificando desempeño y competitividad que podrían fortalecerse a través del entrenamiento, la comunicación interna y estructuras administrativas sólidas, enfocadas en la integración como requisito de supervivencia para la resolución de problemáticas presentadas inicialmente al interior de la entidad y, posteriormente, evaluar mecanismos de prevención para situaciones del entorno, en las que además toma gran relevancia el uso de tecnologías que faciliten la ejecución automática de controles, de manera que se logre consolidar a través de estos la cultura de control que tanto se requiere en este tipo de entidades (Stone, 2014).

En esta vía, las herramientas de control, por más sencillas que sean, son las que en gran medida permiten la integración de las diferentes áreas, procesos y personal, permitiendo que la empresa se direccione a un accionar más eficiente y dinámico frente a los cambios tanto internos como externos.

Al respecto de los controles que se establezcan, es destacable una realidad común, antes mencionada, que se presenta en las MIPYMES y está dada en el sentido de que son los mismos propietarios de la entidad quienes se encargan de diseñar, implementar y monitorear los controles que ellos mismos consideran pertinentes (Castañeda, 2013). Dicha característica genera controversias entre subjetividades del responsable del control interno de la entidad y posibles aspectos o situaciones que requieren miradas más holísticas y objetivas para la resolución de conflictos con proveedores, clientes, colaboradores y, en general, cualquier agente de interés, de tal forma que, en la medida de lo posible, requiere de la participación de varios agentes que propicien espacios de evaluación objetiva e independiente de los procesos aplicados.

En el contexto de las MIPYMES, es válido afirmar que éstas contemplan (sobre todo de manera implícita) algún plan de negocios en el que los propietarios establecen prioridades al menos en el mediano plazo. Dicho modelo a su vez se encuentra sujeto a los cambios que experimenta el mercado en el cual se desenvuelve. Al ser las condiciones del mercado un elemento externo a la empresa se hace necesario ejercer un control en los cambios que puedan resultar en un impacto material para la continuidad de la organización.

No obstante, es claro que la influencia que cualquiera de estas organizaciones pudiese ejercer sobre las condiciones del mercado es casi nula por lo que deben tomar, en su defecto, actitudes proactivas que tiendan a garantizar su estabilidad en el tiempo. Al respecto, Gonzáles (2010) manifiesta que las empresas deben atender al crecimiento en el uso de nuevas tecnologías que facilitan el trabajo y generan resultados mucho más eficientes, modificando constantemente no sólo la forma en la que dichas tecnologías participan en el mercado sino también la forma en cómo se realiza la gestión de la organización con la intermediación de dichas herramientas. Lo anterior permite inferir que en definitiva las nuevas 
realidades económicas conllevan al uso de herramientas que les permitan a empresarios tener un control de los procesos y a su vez faciliten un ajuste de los sistemas de información internos que propicien escenarios para análisis mucho más completos y fidedignos de la realidad organizacional.

Otra parte destacable de la investigación se ha enfocado hacia el estudio de las figuras de vigilancia que pueden recaer sobre los procesos de control que se tengan en la entidad. En este sentido, por ejemplo, toma relevancia la figura del auditor externo que, si bien tiene implícita la responsabilidad de la evaluación del sistema de control interno de la entidad, es muy probable que la mayoría de las organizaciones que cumplen con los criterios para ser consideradas como MIPYMES no estén en la obligatoriedad de tener dicha figura de control y mucho menos probable que la implementen de manera voluntaria, dados los costos que ello acarrea (Bentley, Newton y Thompson, 2017).

En general, han sido muy variados los enfoques que se han tomado para abordar los asuntos de gestión en las MIPYMES, tanto a nivel local como internacional. Sin embargo, es necesario asentar la discusión en torno a los procesos específicos en que se usan los mayores controles que se implementen en una región geográfica y que se den discusiones en torno a ello para alcanzar un nivel de profundización respecto a la implementación de sistemas de control interno y la identificación de oportunidades de mejora que surjan de dicho análisis.

\section{Metodología}

De acuerdo con la información de la Cámara de Comercio del oriente antioqueño, la población total de MIPYMES para la región en el año 2015 fue de 26.627 (ver tabla 1), que representó el 99,6\% del total de empresas ubicadas en esta zona geográfica. En este sentido, para encontrar la cantidad apropiada y representativa de empresas a las que se les aplicaría la encuesta, se utilizó la fórmula de muestreo para poblaciones finitas, considerando un factor de éxitofracaso del $50 \%$ con miras a maximizar el tamaño de esta. Para ello se estableció un error permitido de 5\%, para un nivel de confianza de $95 \%$, lo que dio como resultado una muestra total de 379 entidades. Asimismo, se procedió a tomar el total de MIPYMES por cada municipio del oriente antioqueño y se estratificó la muestra inicialmente obtenida para cada uno de ellos, en consideración a su participación proporcional consolidada en el global de entidades reportadas tal como se muestra en la tabla 1. 
Tabla 1: Estratificación de la muestra

\begin{tabular}{lccc}
\hline \multicolumn{1}{c}{ Municipio } & MIPYMES & Muestra & \% de la Muestra \\
\hline Rionegro & 8861 & 126 & $33.2 \%$ \\
La Ceja & 3168 & 45 & $11.9 \%$ \\
Marinilla & 2458 & 35 & $9.2 \%$ \\
Carmen de Viboral & 2354 & 33 & $8.7 \%$ \\
Guarne & 1880 & 27 & $7.1 \%$ \\
Retiro & 1320 & 19 & $5.0 \%$ \\
Santuario & 1305 & 19 & $5.0 \%$ \\
La Unión & 541 & 8 & $2.1 \%$ \\
San Vicente & 485 & 7 & $1.8 \%$ \\
Guatapé & 446 & 6 & $1.6 \%$ \\
Peñol & 409 & 6 & $1.6 \%$ \\
San Carlos & 365 & 5 & $1.3 \%$ \\
San Rafael & 300 & 4 & $1.1 \%$ \\
Granada & 194 & 3 & $0.8 \%$ \\
Alejandría & 140 & 2 & $0.5 \%$ \\
Concepción & 127 & 2 & $0.5 \%$ \\
Sonsón & 762 & 11 & $2.9 \%$ \\
Abejorral & 413 & 6 & $1.6 \%$ \\
Nariño & 157 & 2 & $0.5 \%$ \\
Argelia & 156 & 2 & $0.5 \%$ \\
Cocorná & 361 & 5 & $1.3 \%$ \\
San Luis & 255 & 4 & $1.1 \%$ \\
San Francisco & 168 & 2 & $0.5 \%$ \\
Población & 26.627 & 379 & $100 \%$ \\
\hline \multicolumn{1}{c}{ Fuente: } & $5 \%$ & \\
\hline
\end{tabular}

Fuente: Elaboración propia con base en información de la Cámara de Comercio del Oriente Antioqueño.

Con base en el número de muestras a evaluar por cada uno de los municipios, se procedió a visitar y aplicar el cuestionario o encuesta a las empresas seleccionadas a través de la estratificación de la muestra (ver tabla 1), el instrumento estuvo compuesto por un total de 42 preguntas (ver anexo 1), en las 379 MIPYMES que permitieron el acceso al equipo investigador. El período en el cual se diligenciaron los cuestionarios fue el año 2016. Dentro del cuestionario se indagó específicamente en seis preguntas orientadas a lo que se puede denominar el control interno contable, dentro de las que se toman las que hacen referencia al uso de tecnologías de la información, pues el uso de 
Castaño, C., Zamarra, J., y Salazar, E. El control interno y las tecnologías de la información en las Mipymes...

estas tecnologías en sí mismo busca la eficiencia en los procesos contables, financieros y de control organizacional.

La validez del cuestionario utilizado para la recolección de la información está dada en términos de los estudios preliminares como Miranda, Castaño y Coronado (2016), Miranda, González y Castaño (2015), Álvarez, López y Mejía (2014), Castaño y Zamarra (2012), Castaño y Correa (2010), Correa, Ramírez y Castaño (2009) y Correa y Jaramillo (2008), y la información base que se tenía sobre las empresas de la región en estudio y sus particularidades, de manera que se enfocaron las preguntas con miras a la obtención de respuestas que fueran razonables y que le permitieran al equipo realizar un análisis completo sobre la situación de dichas empresas en torno a la caracterización de las mismas desde el ámbito contable y financiero.

Para efectos de este texto, y como ya se mencionó, se seleccionaron para la realización de los análisis las preguntas que tienen mayor incidencia en los asuntos relacionados con el control interno y, en general, con las figuras de control que aplican sobre las empresas y, además, sobre las preguntas que se relacionan con el uso de las tecnologías de la información para la optimización de procesos y evaluaciones interna (preguntas 25 a la 30, ver anexo 1). La confiabilidad se verificó con el alfa de Cronbach con un resultado de 0.92 , lo cual es un resultado que da una alta fiabilidad al trabajo y que tiene una alta relación con la consistencia de las respuestas de los encuestados.

Luego de haber efectuado la fase de indagación y recolección de la información en los diferentes municipios se realizaron los análisis sobre los resultados obtenidos. Dichos análisis guardan relación con estudios de tipo exploratorio, dado que se tomaron las respuestas y se tabularon para caracterizar las particularidades de la gestión financiera de las MIPYMES del oriente antioqueño y en este caso en particular, las tendientes al control interno de la organización con un enfoque en el proceso de información financiera.

\section{Resultados}

Sin duda alguna uno de los elementos principales en la determinación y evaluación de cualquier sistema de gestión está dado en torno a la existencia de dicho sistema de gestión. En este caso puntual, la encuesta estuvo orientada a la identificación de la existencia o no de un sistema de control interno dentro de las empresas que hicieron parte de la muestra a las cuales se les aplicó el instrumento, sin importar si dicho sistema estaba o no documentado formalmente.

En ese orden de ideas, se consultó a las MIPYMES por la existencia de controles internos dentro de la entidad, a lo cual $16 \%$ de las respuestas afirmaron tener establecido un sistema de control interno (SCI) documentado, implementado y funcionando en el tiempo. Por su parte, 38\% de los encuestados afirmó sobre la existencia de controles que funcionan regularmente, pero sin tenerlos 
documentados, lo que hace inferir que todo el proceso se hace de manera empírica. Finalmente, $46 \%$ de las respuestas estuvieron orientadas en términos negativos hacia la existencia de algún tipo de control (ver gráfico 1).

Gráfico 1: Existencia del Sistema de Control Interno

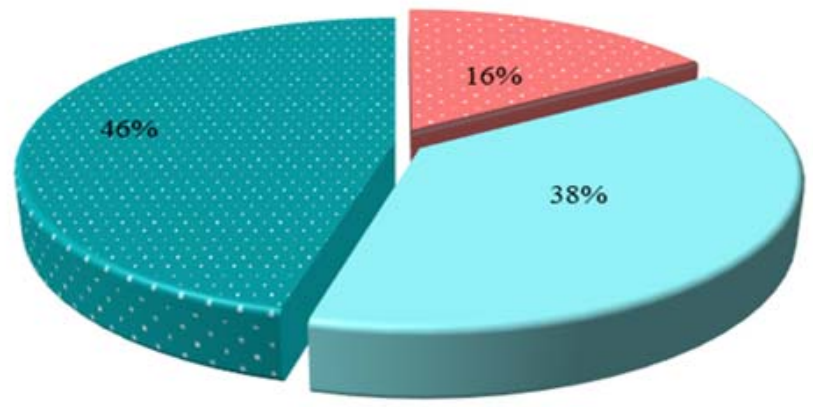

a SI, y está por escrito MSI, pero no está por escrito. aNO

Fuente: Elaboración propia.

Con respecto a estos últimos empresarios que manifiestan no hacer uso de herramientas de control en sus entidades, los mismos han manifestado que la causa principal de la no existencia de controles está soportada en que los propietarios de la organización son los que se encargan de la operación de éstas y no consideran importante establecer controles, puesto que al ser ellos mismos los administradores, no existen riesgos que los puedan afectar desde el punto de vista operativo o financiero, dado un "control directo" que ellos mismos tienen (Leenders y Waarts, 2003).

Esta manifestación deja en evidencia una falencia que de por sí ya era previsible (Speckbacher y Wentges, 2012), en el sentido que representa una oportunidad de mejora importante en cuanto a la cultura del control o, en su defecto, autocontrol que no es característico en las MIPYMES, puesto que, por ejemplo, los empresarios no tienen ningún control ni siquiera manual y periódico sobre procesos básicos que se ejecutan día a día como lo son las compras y las ventas. Estos controles, si bien no requieren de gran experticia por parte del propietario, resultan pertinentes a la hora de evitar obsolescencia de mercancía, desabastecimiento de productos, y demás sucesos que incrementan los costos a causa de una carente toma de decisiones respecto del rumbo y la planeación y evaluación de las actividades.

Este hallazgo denota, por demás, un aspecto que se había mencionado en la contextualización teórica de esta investigación y que hace referencia a la diferencia práctica entre los profesionales de la contabilidad y los administradores de micro y pequeñas empresas que conlleva a que estos últimos no sientan la 
necesidad de recurrir a los servicios profesionales y de asesoría ofrecidos por los expertos. En buena medida la anterior situación puede darse debido a que estos empresarios tienen sus propios mecanismos de administración y no es de su agrado incluir a personas externas en el manejo de la empresa, aun cuando son conscientes de que dichos profesionales poseen los conocimientos y las capacidades para atender de manera satisfactoria e integral las problemáticas que se detecten en la organización.

Otro aspecto importante de indagación estuvo orientado a la determinación de los procesos en los que se tienen establecidos controles internos. En este ítem se evidenció que $43,52 \%$ de los propietarios o administradores encuestados no cuentan en sus empresas con ningún proceso de control interno financiero implementado (ver tabla 2). Los resultados muestran que, en su mayor parte, los controles están establecidos sobre los procesos más básicos de la entidad como lo son las ventas y las compras. Siguiendo esta línea los controles internos sobre procesos como los de inventarios y de nómina han venido tomando fuerza y sobre todo en entidades de tamaño medio en las que tienen mayor aplicabilidad dada una mayor cantidad de productos que se ofertan o un mayor número de personal sobre el que recaen todas las responsabilidades de tipo legal que conlleva todo el proceso de contratación en Colombia.

Tabla 2: Procesos de control interno implementados

\begin{tabular}{lc}
\hline Proceso & $\begin{array}{c}\text { \% aplicación en MIPYMES } \\
\text { del oriente antioqueño }\end{array}$ \\
\hline Compras & $43,78 \%$ \\
Inventarios & $38,86 \%$ \\
Informes financieros & $11,92 \%$ \\
Capital y deuda & $13,47 \%$ \\
Nómina & $29,01 \%$ \\
Ventas & $46,89 \%$ \\
Todos los procesos de control financiero & $0,65 \%$ \\
Ningún proceso de control financiero & $43,52 \%$ \\
\hline
\end{tabular}

Fuente: Elaboración propia.

En este punto es importante aclarar que, dadas las condiciones propias de las empresas, documentar estos procesos requiere un amplio conocimiento sobre el funcionamiento y la estructura de la entidad, que por ser los mismos dueños quienes administran la entidad es una tarea que queda relativamente cubierta en una manera fácil y rápida. No obstante, las características de las empresas y lo evidenciado en la investigación, conlleva a pensar que los controles 
se aplican muy empíricamente y de forma manual, por lo que son escasas las herramientas tecnológicas que se usan para dichos propósitos o los procedimientos de documentación que se tengan sobre los mismos, sobre todo en el contexto de las micro y pequeñas empresas.

Un aspecto importante respecto a ello es que los controles internos de este tipo de empresas están muy enfocados al área operacional como tal. Si bien ello no está mal, es factible mencionar la necesidad de un mayor número de actividades de control encaminadas directamente hacia la vigilancia de los saldos contables de la entidad para una mayor eficiencia de los recursos y la implementación de herramientas de gestión como los presupuestos periódicos y el control sobre la ejecución de estos.

Por su parte, el control contable que recae sobre los procesos financieros en las MIPYMES se ha convertido en un elemento del día a día que opera de manera manual en las empresas micro y las de categoría pequeña empresas se hace de manera automatizada mediante software o herramientas de Office como Excel en las medianas empresas. Lo anterior toma relevancia si se considera que la mayoría de estas empresas solo atienden deberes contables en los casos en que por alguna normatividad especifica o por su tamaño y responsabilidades están obligados a hacerlo y que, en muchos de los casos, ni siquiera cumplen con la totalidad de los requerimientos que le son hechos desde ese punto de vista legal y financiero con importantes consecuencias en materia fiscal por la evasión o elusión que estas entidades pueden realizar (Bedoya y Rua, 2016).

Otro punto a tratar fueron los agentes de control que intervienen en las empresas, respecto a los cuales se obtuvo que $65 \%$ de las empresas encuestadas no poseen un agente de control (ver gráfico 2), bien sea porque no es requerido por la ley o porque son pequeñas organizaciones que no ven la necesidad de tener o implementar estos procesos de seguimiento, por lo cual, las empresas que poseen revisor fiscal es porque están constituidas como un tipo societario que lo requiere (en ningún caso por voluntariedad), mientras que aquellas que tienen alguna figura similar al auditor externo y/o interno, lo tienen principalmente porque son medianas empresas que están constituidas con personería jurídica, por lo cual buscan dar fiabilidad sobre la información contenida en los Estados Financieros, aspecto que logran al tener un mayor control sobre sus operaciones. 
Castaño, C., Zamarra, J., y Salazar, E. El control interno y las tecnologías de la información en las Mipymes...

Gráfico 2: Agentes de control que intervienen

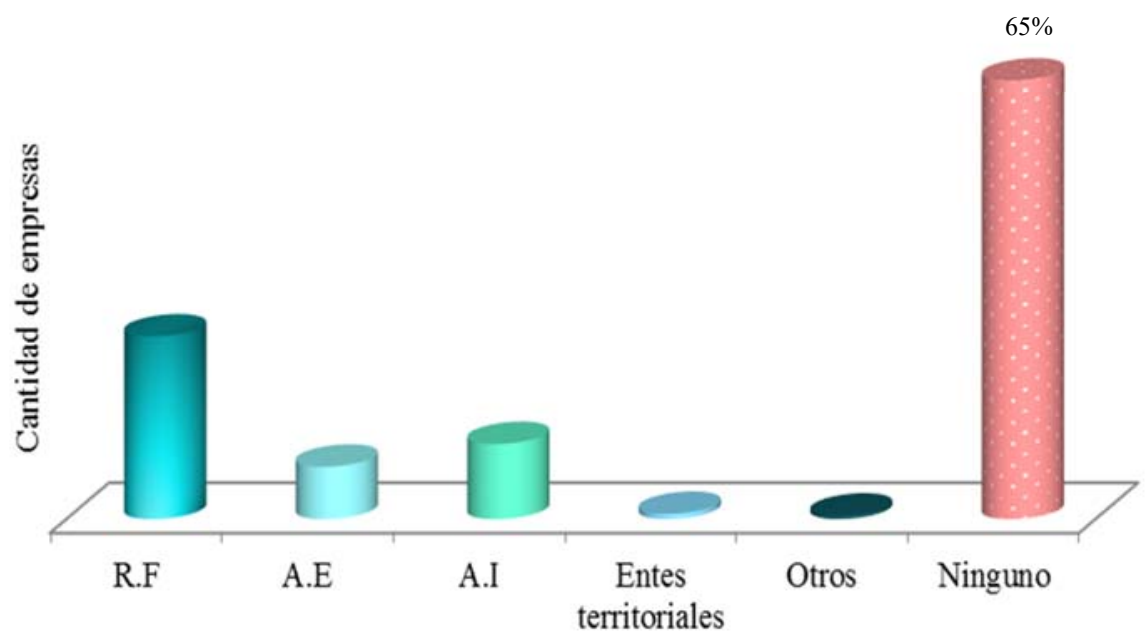

Agentes de control: R.F. Revisor Fiscal A.E. Auditor Externo A.I. Auditor Interno

Fuente: Elaboración propia.

Este último hallazgo tiende a delimitar la existencia de medidas de control en las entidades estudiadas. Sin embargo, su aplicación, como ya se ha mencionado, solo atiende a coyunturas o eventualidades que requieren de la evaluación de personas externas con el conocimiento para ayudar a resolver dicha problemática y que, aun después de identificada dicha situación problema, no es atendida de manera preventiva $a$ posteriori.

Respecto al procesamiento de la información contable y a la necesidad de utilizar herramientas tecnológicas que permitan que esta labor se lleve de manera ordenada dentro de una organización, se indagó acerca de los programas computarizados o software que permiten procesar los datos de la operación contable de las organizaciones, que el $38 \%$ de las empresas no utiliza ningún software, lo que da a suponer que recurren a los antiguos métodos de registro manual en los distintos libros de contabilidad, siendo poco tecnificados. Por su parte, $62 \%$ afirmó tener un software contable y/o administrativo como tal.

El software utilizado se caracteriza por ser muy variado (ver gráfico 3), dentro de los aplicativos tecnológicos más usados se encuentran Contai con un $14 \%$, World Office con un $13 \%$, seguido de SIIGO con un $11 \%$. Sin embargo, no se evidencian tendencias marcadas en la utilización de un software contable específico. Adicionalmente, las microempresas que utilizan algún software en su mayoría utilizan pocos módulos de estos, ya que no tienen un amplio conocimiento acerca de la parametrización de estos. 
Gráfico 3: Software contable utilizado

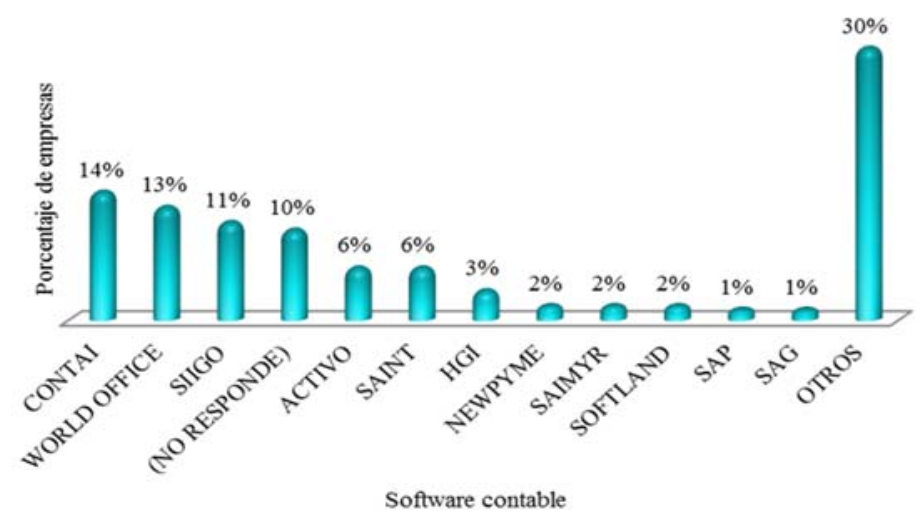

Fuente: Elaboración propia.

En lo que se refiere a las bases de datos, como se observa en el gráfico 4, se encontró que solo $7 \%$ de las empresas encuestadas no cuenta con ninguna base de datos actualizada; es de aclarar que hay empresas que cuentan con varias bases de datos, por lo que el $80 \%$ de estas posee base de datos actualizada de sus clientes, $81 \%$ tiene bases de datos de sus proveedores, $54 \%$ cuenta con información actualizada de su personal, y el 39\% de las empresas identifica a las entidades financieras. Como se observa, la mayoría de las empresas cuentan en promedio con dos bases de datos actualizadas, lo cual es fundamental para tener una adecuada gestión de los distintos procesos de una organización, como las ventas, las compras, la financiación, entre otros, puesto que se facilita la comunicación con terceros.

Gráfico 4: Bases de datos actualizadas

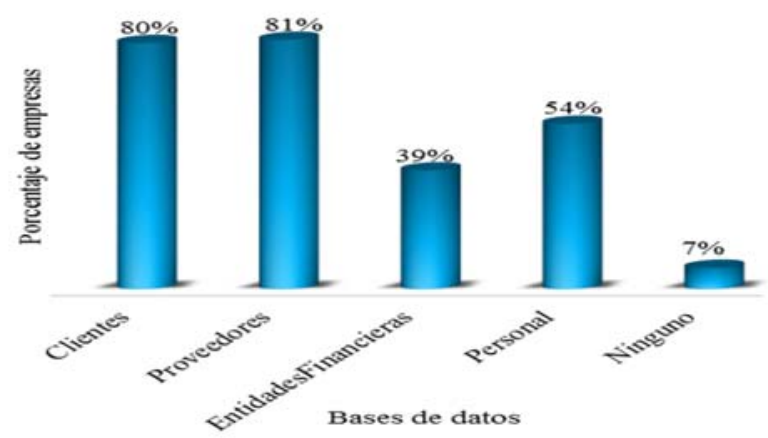

Fuente: Elaboración propia. 
En lo referente a los sistemas de información diferentes a los softwares contables, en el gráfico 5 se observa que un 16\% de las empresas utiliza otros tipos de herramientas dentro de las cuales se encuentra que las más usadas son la información en la Nube con un 6\%, seguido de Excel con $2 \%$ y otros con un $7 \%$; dentro estos se encuentran opciones de respuesta como ERP, Business Intelligence y programas de nómina. Por otro lado, el $84 \%$ restante de las empresas no utilizan otras herramientas tecnológicas, por lo que se observa que no se le otorga gran importancia o que debido al tamaño de las organizaciones no lo consideran de utilidad, ya que gran parte de sus procesos son manuales.

Gráfico 5: Otras herramientas tecnológicas

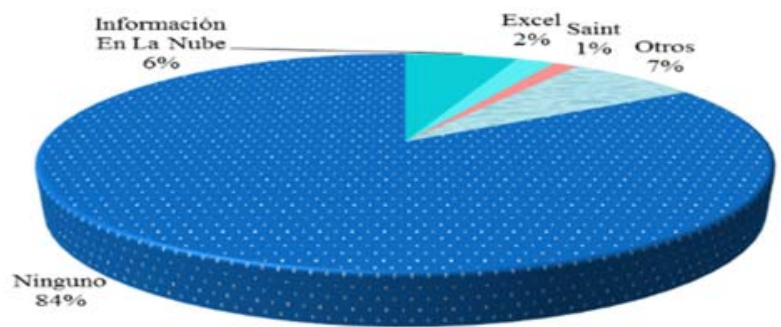

Fuente: Elaboración propia.

Es en este punto donde toma relevancia la integración que pueden hacerse entre los sistemas de información y los controles internos que se implementen en la organización para optimizar el uso tanto de unos como de otros y aprovechar al máximo las oportnidades que brindan estas herramientas de gestión al ser utilizadas con fiabilidad en las MIPYMES de cualquier sector en general.

\section{Conclusiones}

Los análisis descritos anteriormente, permiten inferir que las MIPYMES del oriente antioqueño aún presentan en buena medida oportunidades de mejora en torno a la implementación de sistemas de control interno que les permitan realizar una gestión adecuada de los riesgos a los que se enfrenta la compañía, en búsqueda de una gestión integral de la organización. Lo cierto es que la gestión de riesgos que se hace es poca, incluso en aquellas entidades de mayor tamaño, en las que es estrictamente necesario tener algunos controles, solo se aplican de manera correctiva y no preventiva, de manera que la administración pueda aprovechar recursos importantes en la maximización de resultados y mejoras operacionales en lugar de pasar el tiempo en la resolución de problemas.

Sin embargo, también es válido concluir que dichas herramientas de gestión están ausentes en el sector, debido a un desconocimiento generalizado que afecta en mayor medida a las micro y pequeñas empresas, y en las cuales es 
muy importante generar campañas para incentivar y concientizar sobre las facilidades, beneficios y ventajas competitivas que genera un sistema, aunque sea básico de control interno. Los gerentes, administradores y propietarios de estas entidades deben comprender la importancia de los sistemas de control interno de manera que dimensionen su influencia en el cumplimiento de los objetivos y metas de la organización, así como su incidencia en la determinación de politicas, procedimientos y oportunidades para la empresa.

Adicionalmente, es relevante y rescatable que existan, en buena medida, algunos sistemas de información y gestión contable en las medianas empresas, que faciliten y optimicen la toma de decisiones por parte de la administración. En este sentido, también es importante destacar la influencia que en estas entidades puede tener, el tipo de administración, más aún si está a cargo de los dueños de la entidad, pues no siempre están capacitados adecuadamente para distinguir sus necesidades personales y separarlas de las de la empresa.

La integración entre sistemas tecnológicos y sistemas de control interno tampoco ha resultado eficaz en las empresas encuestadas, puesto que solo en algunos casos de empresas de tamaño mediano, se ha determinado la existencia tanto de sistemas de control como de software o elementos tecnológicos que contribuyan a la gestión conjunta. No obstante, en los casos en los que se dio dicha coincidencia no se integraban ambos sistemas de manera óptima, puesto que ambos procesos eran coordinados de manera independiente.

En concreto, los procesos financieros y de control interno que afrontan las MIPYMES del oriente antioqueño son muy similares a las del resto del país y, en general, a las empresas de dicho tamaño en el mundo. Sus riesgos, problemas, desventajas y oportunidades de mejora se mantienen, más aún cuando las gestiones son desorganizadas y desenfocadas, esto se convierte en una de las principales causas de la disolución y liquidación anticipada de estas organizaciones.

El logro de un desempeño deseado y, por lo tanto, de una mayor competitividad no puede quedar supeditado a la existencia o no de un sistema de control, pero no puede negarse la importancia que el mismo tiene para el alcance de los objetivos de corto, mediano y largo plazo. Hay claras ventajas en su utilización e implementación en el tiempo, que van desde la generación de una cultura de cumplimiento y transparencia hasta la optimización de las decisiones que son tomadas en la dirección de la entidad, bajo información confiable y razonable sobre la gestión que se ha hecho y los problemas que en el camino van surgiendo y se van solucionando, con miras a la consecución de sus objetivos y la sostenibilidad de la MIPYME en el tiempo.

Existen múltiples oportunidades de investigación respecto a estos temas de control interno y figuras de control, más aún si se enfocan en las MIPYMES, en cuanto a las oportunidades de mejora para la consolidación de procesos confiables que contribuyan al fortalecimiento del sector y permitan ahondar en las 
consideraciones y discusiones investigativas en pro de la mejora continua de las herramientas que facilitan la toma de decisiones en las organizaciones. Así mismo, es necesario revisar la articulación entre las políticas públicas y la gestión de las MIPYMES, pues esta falta de integración a llevado a la ineficacia del uso de recursos públicos en actividades que finalmente no logran los objetivos inicialmente planteados (Quintanilla-Ortiza y Díaz-Jiménez, 2019).

Por último, se establecen retos para la formación de contadores públicos comprometidos con las organizaciones de la región y que entiendan las situaciones del contexto latinoamericano (Rojas, 2017) y en procura de información que trascienda lo meramente financiero y considere la importancia de la sostenibilidad en el largo plazo sin importar el tamaño organizacional (Bernal y Santos, 2018).

\section{Anexos}

\section{Anexo 1. Cuestionario}

Caracterización de las prácticas de gestión contable y financiera de las MIPYMES del Oriente Antioqueño

El objetivo de esta encuesta es identificar las principales características de las prácticas de gestión contable y financiera de las MIPYMES del Oriente Antioqueño. Este estudio es realizado por un grupo de Investigadores de la Facultad de Ciencias Contables de la Universidad de Antioquia; agradecemos su colaboración, respondiendo las siguientes preguntas.

El contenido de esta encuesta solo se utilizará con fines académicos objeto de esta investigación y serán de absoluta confidencialidad. Los resultados obtenidos serán enviados al correo electrónico que aparezca diligenciado en el formato.

1. Nombre de la empresa:

2. Seleccione el sector al que pertenece la empresa

Industrial $\square$

Comercial $\square$

Servicio $\square$

Sub - sector:

3. Cargo del responsable del Cuestionario:

4. Página web y/o correo electrónico de la empresa:

5. Municipio donde está ubicada la empresa:

6. Valor de los activos del último año (a Diciembre 31 de 2015) 
SMMLV: Salario Mínimo Legal Mensual Vigente

Hasta 500 SMMLV (\$ 322.175.000)

Entre 501 - 5.000 SMMLV (\$ 322.819.350 - \$ 3.221.750.000)

Entre 5001 - 30.000 SMMLV (\$ 3.222.394.350 - \$ 19.330.500.000)

$\square$ Más de 30.000 SMMLV

7. Número de empleados de la empresa (a Diciembre 31 de 2015)
$\square$ Entre $1-10$
Entre $11-50$
$\square$ Entre $51-200$
$\square$ Más de 200

8. Ingresos del último año (a Diciembre 31 de 2015)

Hasta 6.000 SMMLV (\$ 3.866.100.000)

$\square$ Más de 6.000 SMMLV

9. ¿Cuántos años lleva funcionando su empresa?

$\square$ Entre 0 - 3 años

Entre $4-6$ años

Entre 7 - 10 años

$\square$ Más de 10 años

\section{Formalización}

10. Su empresa funciona como:

$\square$ Persona natural

Empresa unipersonal

$\square$ Entidad Sin Ánimo de Lucro

Sociedad comercial

\begin{tabular}{|l|l|}
\hline Tipo de Sociedad Comercial & \\
\hline Sociedad colectiva & \\
\hline Sociedad de responsabilidad limitada & \\
\hline Sociedad anónima & \\
\hline Sociedad en comandita simple & \\
\hline Sociedad en comandita por acciones & \\
\hline Sociedad por acciones simplificada & \\
\hline
\end{tabular}

\section{Organizacional}

11. ¿Cuenta con una estructura organizacional definida (organigrama o algo similar)?<smiles>ONC1CCC1</smiles>

12. En caso de que la respuesta anterior sea afirmativa, ¿la empresa identifica el área contable o financiera dentro de esta estructura?

$\square$ SI $\square$ NO 
Castaño, C., Zamarra, J., y Salazar, E. El control interno y las tecnologías de la información en las Mipymes...

13. De los siguientes elementos seleccione los que están contemplados en la empresa formalmente (por escrito)
$\square$ Misión
Visión
Objetivos
Valores
Manual de funciones y procedimientos
Plan Estratégico
Flujogramas

14. ¿La empresa cuenta con procesos de planeación?

$\square$ SI $\square$ NO

15. Si la respuesta anterior es afirmativa, seleccione los plazos establecidos para estos.

$\square$ Corto plazo (Menos de 1 año)

$\square$ Mediano plazo (Entre 1 y 3 años)

$\square$ Largo plazo (Más de 3 años)

16. ¿Cuál es el nivel de estudios del administrador de la empresa?

$\square$ Primaria

Secundaria

Tecnológica ¿Cuál?

$\square$ Profesional ¿Cuál?

$\square$ Especialización ¿Cuál?

$\square$ Maestría ¿Cuál?

$\square$ Doctorado ¿Cuál?

17. ¿Tienen identificados los principales competidores de su empresa?

$\square$ SI $\square$ NO

\section{Gestión contable}

\section{Informes contables}

18. ¿Cuáles Estados Financieros se elaboran actualmente en la empresa?

$\square$ Estado de Resultados

\section{Balance General}

Estado de Cambios en la situación Financiera

Estado de Flujos de Efectivo

$\square$ Estado de Cambios en el patrimonio

$\square$ Notas a los estados financieros

$\square$ Ninguno de los anteriores

19. ¿Cuál es la periodicidad de los Estados Financieros?

$\square$ Mensual

$\square$ Trimestral

$\square$ Semestral 
$\square$ Anual

Otra ¿Cuál?

20. ¿Qué respaldo tienen los estados financieros frente a terceros?

$\square$ Certificados

$\square$ Dictaminados

$\square$ Ninguno

21. ¿Qué fines persigue la elaboración de estados financieros?

Cumplimiento legal

$\square$ Gestión administrativa

$\square$ Acceso a la financiación

$\square$ Acceso a inversión

$\square$ Responsabilidad social

22. ¿Qué otros informes contables elabora, diferentes a los estados financieros?

Informes de gestión

Informes social

$\square$ Informes de sostenibilidad

Informes estratégicos de control de gestión

$\square$ Otros ¿Cuál?

23. ¿Qué forma de contratación tiene el Contador Público de la empresa?

Tiempo completo

Medio tiempo

$\square$ Prestación de servicios - tiempo parcial

$\square$ No tiene contador

24. ¿Cuenta la empresa con políticas establecidas para la presentación de informes internos?

$\square$ SI $\square$ NO

¿Cuáles?

\section{Control Interno Contable}

25. ¿La empresa cuenta con un sistema de control interno establecido?

$\square$ SI $\quad \square$ NO

26. En caso de que la respuesta anterior sea afirmativa ¿En qué tipo de procesos contables y financieros es utilizado este sistema?

$\square$ Ventas
$\square$ Compras
$\square$ Inventarios
$\square$ Nómina
$\square$ Capital y deuda
$\square$ Informes financieros


Castaño, C., Zamarra, J., y Salazar, E. El control interno y las tecnologías de la información en las Mipymes...

27. ¿Qué agentes de control intervienen en la empresa?

$\square$ Auditor externo

Auditor interno

Revisor Fiscal

$\square$ Ninguno de los anteriores

Tecnologías de la información

28. ¿Utiliza la empresa algún software de procesamiento de información contable?

$\square$ SI

¿Cuál?

29. ¿Cuenta con bases de datos actualizadas de?

$\square$ Clientes

Proveedores

$\square$ Entidades Financieras

$\square$ Personal

Otros

30. ¿Qué otro tipo de tecnologías utiliza la empresa en sus procesos contables y financieros? Por ejemplo: Información en la nube, ERPs, Bussines Inteligent, Big data, entre otros

\section{Gestión financiera}

31. ¿Cuáles de las siguientes herramientas son utilizadas por la empresa para la gestión financiera?

$\square$ Presupuestos

Cuadro de mando integral

Planeación financiera

Indicadores financieros

Análisis estructural de los estados financieros

Cálculo del costo de capital

Análisis del capital de trabajo

Evaluación de proyectos

Matemáticas financieras

Sistema de costos

Benchmarking

Otros ¿Cuáles? 
32. Marque con una $\mathrm{X}$, ¿Con qué frecuencia se actualizan y analizan estas herramientas?

Presupuestos

Mensual Bimestral Trimestral Semestral Anual

Cuadro de mando integral

Planeación financiera

Indicadores financieros

Análisis estructural de EEFF

Cálculo del costo de capital

Análisis del capital de trabajo

Evaluación de proyectos

Matemáticas financieras

Sistema de costos

Benchmarking

Otros

33. Si tiene sistemas de indicadores financieros, ¿cuáles indicadores utilizan en la compañía?

$\square$ Razón corriente
$\square$ Prueba Ácida
$\square$ Rotación de Cuentas por Cobrar
$\square$ Rotación de Inventarios
$\square$ Rotación de Cuentas por Pagar
$\square$ Nivel de endeudamiento
$\square$ Rentabilidad del activo
$\square$ Rentabilidad del Patrimonio
$\square$ Márgenes de utilidad
$\square$ EBITDA
$\square$ Margen EBITDA
$\square$ EVA
$\square$ Otros ¿Cuáles?

34. Si calcula el EVA, ¿se ha generado valor en los últimos dos años?

$\square$ SI $\square$ NO

35. Organice los siguientes medios de financiación de los más utilizados a los menos utilizados. (1 más utilizado, 6 menos utilizado). No enumere aquellas que no utiliza.

$\square$ Crédito con el sistema financiero

Apoyo del Gobierno

Crédito Informal

Capital propio (aportes nuevos) 
Castaño, C., Zamarra, J., y Salazar, E. El control interno y las tecnologías de la información en las Mipymes...

Generación interna de fondos (utilidades)

Créditos con los proveedores

36. ¿La empresa ha contratado servicios de asesoría y/o consultoría en temas contables y/o financieros?

$\square$ SI $\quad \square$ NO

¿En qué temas particulares se han contratado las asesorías y/o consultorías?

\section{Proceso de toma de decisiones}

37. ¿Dentro de la estructura organizacional está claramente definido quiénes son los principales tomadores de decisiones en temas como inversión, financiación y distribución de utilidades o excedentes?

$\square$ SI ¿Quiénes?

$\square$ NO

38. ¿Se utilizan los informes contables y financieros en el proceso de toma de decisiones?

$\square$ SI ¿En qué decisiones?

$\square$ NO

39. ¿La información proporcionada por el sistema de control interno (en caso de que este exista), es utilizada en la toma de decisiones?

$\square$ SI ¿En qué decisiones?

40. Si la empresa realiza presupuestos y proyecciones, ¿Qué beneficios le ha generado?

41. Ordene de acuerdo a su nivel de importancia para la toma de decisiones los siguientes estados financieros, (1 más importante, 5 menos importante).

Estado de Resultados

Balance General

Estado de Flujos de Efectivo

$\square$ Estado de Cambios en el patrimonio

Notas a los estados financieros

42. ¿Se tiene en cuenta el costo de oportunidad para tomar decisiones sobre el financiamiento de la empresa?

$\square$ SI $\square$ NO. 


\section{Referencias bibliográficas}

Álvarez Orozco, N., López Ramírez, C., y Mejía Serna, P. Y. (2014). Los Retos de las Mipymes Rionegreras frente a la Gestión Financiera Integral, para la toma de decisiones y Continuidad Empresarial a Partir del Modelo de Gestión Financiera Integral para Mipymes. Adversia (14), 1-24. Recuperado de: http://aprendeenlinea.udea.edu.co/ revistas/index.php/adversia/article/viewFile/20902/17493

Alonso, J.; Saraite, L.; Haro de Rosario, A.; Caba-Pérez, C. (2016). Sector bancario a nivel mundial y los factores que influyen en su información sobre responsabilidad social corporativa. Contaduría Universidad de Antioquia, 68, 213-233.

Aguirre, R., y Armenta, C. (2012). La Importancia del Control Interno en las Pequeñas y Medianas Empresas en México. Revista El Buzón de Pacioli (76), 1-17.

Arango,A. ( 2019). MiPymes representan más de $90 \%$ del sector productivo nacional y generan el $80 \%$ del empleo en Colombia. Ministerio de Trabajo de Colombia. Recuperado de: https:/www.mintrabajo.gov.co/prensa/comunicados/2019/septiembre/mipymesrepresentan-mas-de-90-del-sector-productivo-nacional-y-generan-el-80-del-empleoen-colombia-ministra-alicia-arango

Balcazar, A. (2018). Gobernanza corporativa, una propuesta para el mejoramiento en la gestión administrativa y financiera en el hospital E.S.E Nuestra Señora del Carmen Tabio - Cundinamarca. Contaduría Universidad de Antioquia, 73, 13-32. Doi: https:// doi.org/10.17533/udea.rc.n73a01

Bardhan, I., Lin, S., \& Wu, S. (2015). The quality of internal control over financial reporting in family firms. Accounting Horizons, 1(29), 41-60.

Barney, J. (1991). Firm resources and sustained competitive advantage. Journal of Management (17), 199-220.

Bedoya, O. H. y Rua,W. (2016). El concepto de evasión y elusión en términos de la Corte Constitucional, el Concejo de Estado y la Administración de Impuestos y Aduanas Nacionales. Contaduría Universidad De Antioquia, (69), 69-97. Recuperado a partir de https://revistas.udea.edu.co/index.php/cont/article/view/328431

Bentley, K., Newton, N., \& Thompson, A. (2017). Business Strategy, Internal Control over Financial Reporting, and Audit Reporting Quality. Auditing: A Journal of Practice and Theory, 36(4), 49-69.

Bernal, M.; Santos, E., (2018). Una mirada a la contabilidad ambiental. Postulados y retos. Contaduría Universidad de Antioquia, 73, 199-209. Doi: https://doi.org/10.17533/ udea.rc.n73a08

Cámara de Comercio del Oriente Antioqueño. (Agosto de 2015). Análisis Comparativo Empresarial y Regional (ACER). Recuperado de: http://www.ccoa.org.co/contenidos/ categorias/ crecimiento _ regional.php

Castañeda, L. (2013). Los sistemas de información contable una herramienta para la gestión integral de las Pymes. Revista de la Agrupación Joven Iberoamericana de Contabilidad y Administración de Empresas (AJOICA) (11), 31-40.

Castaño Rios, C. E. y Correa García, J. A. (2010). Reflexiones sobre la gestión financiera integral en MIPYMES. Colombian Accounting Journal, 4, 125-147. 
Castaño, C., Zamarra, J., y Salazar, E. El control interno y las tecnologías de la información en las Mipymes...

Castaño Rios, C. E. y Zamarra Londoño, J. E. (2012). Gestión financiera en las MIPYMES colombianas: diagnóstico y propuestas de mejoramiento en escenarios de competitividad global. En XIII Asamblea General de ALAFEC, 9 al 12 de octubre. Buenos Aires, Argentina.

Castaño, C. E., Zamarra, J. E. y Salazar, E. J. (2017). Caracterización de la gestión contable y financiera en Mipymes del oriente antioqueño (Colombia). Escenarios: Empresa y Sociedad, 6 (8), pre-print.

Cesaroni, F., \& Sensuti, A. (2017). Accountants' Role in the Management of Succession: Empirical Evidence from Italy. Journal of Family Business Management, 3(6), 270-290.

Choe, J. (1996). The relationships among performance of accounting information systems, influence factors, and evolution level of information systems. Journal of Management Information Systems, 4(12), 215-239.

Correa García, J. A., Jaramillo Betancur, F. (2008). Modelo de gestión financiera integral para PYMES: prospectiva en escenarios de competitividad global. Proyecto de investigación. Facultad de Ciencias Económicas Universidad de Antioquia.

Correa García, J. A., Ramírez Bedoya, L. J. y Castaño Ríos, C. E. (2009). Modelo de gestión financiera integral para MIPYMES en Colombia. Contaduría Universidad de Antioquia, (50), 187-201. Recuperado de: https://aprendeenlinea.udea.edu.co/revistas/index. php/cont/article/viewFile/16343/14176

Correa, J., Ramírez, L., y Castaño, C. (2010). La importancia de la planeación financiera en la elaboración de los planes de negocio y su impacto en el desarrollo empresarial. Revista Facultad de Ciencias Económicas: Investigación y Reflexión, XVIII(1), 179-194.

Deshmukh. (2004). A conceptual framework for online internal controls. Journal of Information Technology Management, 4(15), 23-32.

Doyle, J., Ge, W., \& McVay, S. (2007). Accruals quality and internal control over financial reporting. The Accounting Review, 5(82), 1141-1170.

Efferin, S., \& Hartono, M. (2015). Management Control and Leadership Styles in Family Business: An Indonesian Case Study. Journal of Accounting \& Organizational Change, 1(11), 130-159.

European Commission. (2009). Overview of family-business-relevant issues: Research, networks, policy measures and existing studies. Final report of the expert group, 1-33.

Fernández, Z., \& Nieto, M. (2005). Internationalization strategy of small and medium-sized family businesses: Some influential factors. Family Business Review, 1(18), 77-89.

Forseilles, S. (2013). Expo MIPYME Digital. Recuperado de: http:/www.easyfairs.com/ es/events_216/expo-mipyme-digital-caribe2013_48673/expo-mipyme-digitalcaribe-2013 48674/

Fuentes-Doria, D, Toscano-Hernández, A, Díaz-Ballesteros, J, y Escudero-Garrido, Y. (2019). Ética y sostenibilidad en la gestión contable: Un análisis documental en el contexto Mundial en los periodos 2009-2018. Contaduría Universidad de Antioquia, 75, 43-67. Doi: https://doi.org/10.17533/udea.rc.n75a02

Giraldo Ruiz, C., Sinisterra Rincón, E., \& Arteaga Londoño, M. (2018). Prácticas contables generalmente utilizadas en el sector informal: Caracterización de las microtiendas 
de la comuna cuatro de Medellín. Contaduría Universidad De Antioquia, (72), 59-80. https://doi.org/10.17533/udea.rc.n72a04

Gómez, L., Cruz, C., Berrone, P., \& De Castro, J. (2011). The Bind that Ties: Socioemotional Wealth Preservation in Family Firms. The Academy of Management Annals, 5(1), 653707.

Gómez, Y., Pérez, R., \& Ramirez, Y. (2017). Guía de mejores prácticas de control interno en las microempresas. Recuperado de: https://usbcalivirtual.edu.co/portal/biblioteca-digital/

Gómez, J., Villarreal, M. y Villarreal, F. (2015b). Las incapacidades de aprendizaje organizacional y su relación con los estilos de aprendizaje organizacional en la Mipyme. Revista Contaduría y Administración, 60(3), 686-702

Gonzáles, P. (2010). Avances y desafíos sobre la enseñanza de la contabilidad de gestión en Colombia desde una perspectiva de mipymes y de la educación superior. Revista Universo Contábil, 3(6), 140-155.

González,L., Viga, C. y Fierro, S. (2017). Prospección Del Riesgo Operativo De Las Mipymes En Colombia. Suma Negocios, 8(18)79-87.

Henri, J. (2006). Organizational culture and performance measurement systems. Accounting, Organizations and Society (31), 77-103.

La República (de agosto de 2019). Mipymes representan 96\% del tejido empresarial y aportan 40\% al PIB. La república. Recuperado de: https://www.larepublica.co/economia/ mipymes-representan-96-del-tejido-empresarial-y-aportan-40-al-pib-2903247

Leenders, M., \& Waarts, E. (2003). Competitiveness and Evolution of Family Businesses: The Role of Family and Business Orientation. European Management Journal, 6(21), 686-697.

Li, Y., Liu, Y., Duan, Y., \& Li, M. (2008). Entrepreneurial orientation, strategic flexibilities and indigenous firm innovation in transitional China. International Journal of Technology Management (41), 223-246.

López, Y.; Venereo, N.; Albuerne, M.; Casas, Y. (2016). Administración financiera del capital de trabajo en la empresa mixta Havana Club International S.A. Contaduría Universidad de Antioquia, 68, 255-285.

Lozano, M. (2015). Strategic decisions of family firms on cash accumuation. RAE: Revista de Administração de Empresas, 55(4), 461-466.

Miranda Torres, J. P., Castaño Rios, C. E. y Coronado Rincón, O. (2016). Pequeñas empresas productoras de alimentos de Navojoa y su gestión financiera. VI Congreso de investigación financiera FIMEF, 25 y 26 de agosto. Guadalajara: México.

Miranda Torres, J. P., González Velásquez, L. M. y Castaño Ríos, C. E. (2015). Diagnóstico de la gestión financiera integral en las Mipymes de Navojoa, Sonora. Obtenido de XIX Congreso internacional de investigación en ciencias administrativas.

Monge, R., Alfaro, C. y Alfaro, J. (2005). TICs en las PYMES de Centroamérica: impacto de la adopción de las tecnologías de la información y la comunicación en el desempeño de las empresas (Primera ed.). Costa Rica: International Development Research Centre.

Pereira, R. (2015). Análise do sistema de controle interno no Brasil: objetivos, importância e barreiras para sua implantação. Revista Contemporânea de Contabilidade., 12(25), 159-188. 
Castaño, C., Zamarra, J., y Salazar, E. El control interno y las tecnologías de la información en las Mipymes...

Quintanilla-Ortiz, D. y Díaz-Jiménez, M. (2019). Una revisión conceptual y de aplicación de la contabilidad de gestión en el sector público. Contaduría Universidad de Antioquia, 74, 35-57. Doi: https://doi.org/10.17533/udea.rc.n74a02

Rivera-Godoy, J. A., y Rendón-Perea, J. D. (2019). Sector avícola en Colombia: rendimiento contable y EVA. Contaduría Universidad De Antioquia, (74), 127-151. https://doi. org/10.17533/udea.rc.n74a06

Romero, F., Melgarejo, Z., Vera, M. (2015). Fracaso empresarial de las pequeñas y medianas empresas (pymes) en Colombia. Suma Negocios. 6(13), 29-41.

Rojas, W. (2017). Asimilación y rupturas de la educación de la contaduría pública: Desde el sur y para el sur. Contaduría Universidad de Antioquia, 71, 85-101

Saha, A., \& Mondal, K. (2012). Internal control policy in the RMG sector of Bangladesh. Asian Business Review (1), 67-71.

Sonrensen, J. (2002). The strength of corporate culture and the reliability of firm performance. Administrative Science Quarterly, 1(47), 70-91.

Speckbacher, G., \& Wentges, P. (2012). The Impact of Family Control on the Use of Performance Measures in Strategic Target Setting and Incentive Compensation: A Research Note. Management Accounting Research, 1(23), 34-46.

Stone, M. (2014). The new (and ever-envolving) direct and digital marketing ecosystem. Journal of direct, data and digital marketing practice (16), 71-74. 